\title{
Toll-like receptors 2 and 6 mediate apoptosis and inflammation in ischemic skeletal myotubes
}

Hemanshu Patel ${ }^{1}$, Cissy Yong ${ }^{1}$, Ali Navi ${ }^{1}$, Sidney G Shaw ${ }^{2}$, Xu Shiwen ${ }^{3}$, David Abraham ${ }^{3}$, Daryll M Baker ${ }^{1}$ and Janice CS Tsui ${ }^{1}$

1. Division of Surgery \& Interventional Science, University College London, Royal Free Campus, London, UK

2. Department of Clinical Experimental Research, University of Bern, Bern, Switzerland

3. Centre for Rheumatology \& Connective Tissue Disease, University College London, Royal Free Campus, London, UK

\section{Introduction}

Peripheral artery disease (PAD) is a major cause of morbidity and mortality and is caused by the accumulation of atherosclerotic plaques within the lower limb arteries. Critical limb ischaemia (CLI) results from progression of this disease leading to chronic muscle ischaemia with tissue inflammation, necrosis and apoptosis.(1) Patients with CLI present with rest pain with or without ulcers or gangrene. At this stage limb salvage requires surgical or endovascular attempts to improve blood flow, however in some patients revascularisation is not possible. An increasing number of studies show that even successful revascularisation does not always lead to improvements in symptoms, functional status or quality of life. $(2,3)$ Indeed $34 \%$ of patients with CLI undergo major limb amputation within 12 months.(4) The lack of improvement in symptoms in patients despite revascularisation is due in part to a myopathy (5-8) within the ischaemic muscle. The pathophysiology of skeletal muscle damage following ischaemia is unclear but a number of detrimental physiological and biochemical changes have been identified. However the mechanism of how hypoxia/ischaemia activates the damaging pathways in muscle are yet to be elucidated.

Toll-Like receptors (TLRs) are pathogen recognition receptors (PRR) of the innate immune system. They are type 1 membrane glycoproteins that contain an external ligand-binding leucine-rich repeat (LRR) motif and a cytoplasmic signalling domain that is similar to the Interleukin-1 (IL-1) receptor and thus termed the Toll/IL-1 receptor (TIR) domain. So far 11 TLRs (TLR 1-11) are thought to be functional in humans and despite their documented role in innate immunity they are widely expressed by both immune and non-immune cells including skeletal muscle (9). TLRs recognise and are activated by pathogen associated molecular patterns (PAMPs) which are microbial molecules that alert the host cell of the presence of invading organisms such as bacteria and viruses. In addition to PAMPs, TLRs are also stimulated by host derived molecules (10) that are released during tissue injury. Endogenous ligands such as high mobility group box 1 protein (HMGB-1) (11), heat shock 
proteins (HSPs) and hyaluronan (12) have been implicated in ischaemia-induced organ damage.

Ligand binding by PAMPs or endogenous molecules leads to TLR dimerization which contributes to ligand specificity. All TLRs form homodimers except TLR1, 2 and 6: TLR2 can heterodimerise with TLR1 or 6 (13) depending on the stimulating ligand. Exogenous or endogenous ligand stimulation of the TLR signalling pathways, of which there are two distinct types, leads to activation of transcription factors such as nuclear factor KB (NF-KB), activator protein 1 (AP-1) and the interferon regulatory factors. This in turn leads to upregulation of pro-inflammatory cytokines such as tumour necrosis factor $\alpha$ (TNF- $\alpha$ ), interleukins 1 and 6 (IL-1 \& 6) and type 1 interferons (IFN). Further, TLR activation has been reported to directly cause apoptosis via Fas-associated death domain protein (FADD) and caspase 8 (14).

Signal transduction following TLR stimulation is dependent upon the TIR domain binding to an adapter protein. There are four adapter proteins that are involved: myeloid differentiation primary response protein 88 (MyD88), TIR domain-containing adapter protein (TIRAP/Mal), TIR domain-containing adapter inducing IFN $\beta$ (TRIF) and TRIF-related adapter molecule (TRAM) (15). The two distinct TLR signalling pathways are the MyD88 dependent and the MyD88 independent pathways whose main adapter proteins are MyD88 and TRIF respectively. All TLRs signal via the MyD88 dependent pathway apart from TLR3 which signals via the MyD88 independent pathway, whilst TLR 4 can signal via either signalling mechanism. The subsequent propagation of the signalling pathway involves the interleukin-1 receptor associated kinase (IRAK) and MAP kinase family. In the TLR signalling pathway the quiescent NF-KB is sequestered by the inhibitor of NF-KB (IKB) protein. Following TLR stimulation and signal transduction, IKB protein is phosphorylated and polyubiquitylated which releases NF-KB to translocate to the nucleus and activate gene transcription.

There is emerging evidence suggesting that TLRs play an important role in ischaemia and ischaemia/reperfusion induced tissue damage in the kidneys, liver, central nervous and cardiovascular systems (16). In particular TLR2 and 4 activation has been strongly associated with ischaemia-induced organ damage $(17,18)$. TLR2 and 4 knockout mice have been shown to be protected against ischaemia-induced liver (19), renal (20) and myocardial (21) injury. Further studies have shown that this protective effect is associated with a reduction in proinflammatory cytokine expression (22) and NF-kB nuclear translocation (21). Despite this strong association between TLRs and ischaemia-induced pathogenesis, very little work has focused on the role that TLRs play in skeletal muscle damage in CLI.

Current treatments for $\mathrm{CLI}$ are aimed at improving blood flow. However there is significant evidence to suggest that this does not always help improve patient symptoms. We have 
therefore taken a novel approach of studying the affected end organ, i.e. the skeletal muscle itself in order to understand whether TLR2 and TLR6 play a role in the ischaemiainduced muscle damage. A better understanding of this pathological process may identify potential therapeutic targets that may be used in conjunction with revascularisation.

\section{Methods}

\section{Human gastrocnemius muscle biopsy}

Open gastrocnemius muscle biopsies were obtained from patients undergoing perigenicular amputation for $\mathrm{CLI}$ and non-ischaemic control samples were obtained from patients undergoing great saphenous vein harvesting for coronary artery bypass grafting. Twelve patients from each group were biopsied. The biopsy is taken as an excisional biopsy rather than using a biopsy needle. Thus the sample size of the biopsy is approximately $4 \mathrm{~mm}$ by $4 \mathrm{~mm}$. At least 3 biopsies were taken from the patient with PAD whilst 1 sample per patient was taken from the control patients. The sample is taken from the medial head of the gastrocnemius muscle and any areas with macroscopic necrosis were avoided. To ensure that none of the control patients had flow limiting PAD, all patients with an ankle-brachial pressure index $(\mathrm{ABPI})$ of $<0.9$ were excluded from the control group. In addition any patient with an infected ulcer or trauma were also excluded from the sample collection. There was no significant difference in the age related risk factors between the 2 groups (Figure 1a). We have previously shown that hypoxia-inducible factor $1 \alpha$ a marker of hypoxia is elevated in muscle samples from patients with CLI compared to control (23). The same technique was used to harvest the muscle samples in both groups and the tissue was snap frozen in liquid nitrogen and stored at $-80^{\circ} \mathrm{C}$ until use.

\section{Cell cultures}

The C2C12 mouse myoblast cell line (European Collection of Cell Cultures (ECACC) no. 91031101, passage no. 13, Salisbury, UK) was plated onto 12-well culture plates (BD Falcon $^{\text {TM }}$, cat. no. 351143, Flintshire, UK) or 8-well chamber slides (BD Falcon, cat. No. 354108, Flintshire, UK) and cultured in Dulbecco's modified Eagle's medium (DMEM, Gibco, Paisley, Scotland) supplemented with $10 \%$ fetal calf serum, penicillin $(100 \mathrm{U} / \mathrm{mL})$, streptomycin $(100 \mu \mathrm{g} / \mathrm{mL})$ and amphotericin B (25 $\mu \mathrm{g} / \mathrm{mL}$, all from Gibco). At $80 \%$ confluence, cells were switched to differentiation medium containing DMEM with $2 \%$ horse serum penicillin $(100 \mathrm{U} / \mathrm{mL})$, streptomycin $(100 \mu \mathrm{g} / \mathrm{mL})$ and amphotericin $\mathrm{B}(25 \mu \mathrm{g} / \mathrm{mL}$, all from Gibco). Differentiation was confirmed by changes in morphology (supplementary 
Figure 1) as well changes in the expression of myogenin protein. Myogenin is preferentially expressed in skeletal myotubes but not myoblasts (23). Following differentiation, usually at day 7-8, myotubes were exposed to simulated ischaemia or normoxia with or without relevant pre-treatments.

\section{Simulated ischaemia}

We have developed a model of simulated ischaemia that has previously been described (23). Chronic ischaemia is difficult to replicate in vitro, however the aim of the simulated ischaemia model was to create an in vitro environment that matched the parameters of chronic ischaemia such as $\mathrm{PO}_{2}, \mathrm{CO}_{2}, \mathrm{pH}$, apoptosis and lactate dehydrogenase release which have been demonstrated in skeletal muscle of patients with CLI. Briefly, 24 hours following culture media change, myotubes (in open dishes) were exposed to $20 \% \mathrm{CO} 2+80 \% \mathrm{~N} 2$ (British Oxygen, Luton, UK) in hypoxic chambers (Modular Incubator Chamber, MIC-101; Billups-Rothenberg, Del Mar, CA, USA). The chamber was flushed with gas at $10 \mathrm{l} / \mathrm{min}$ for $20 \mathrm{~min}$ and then sealed. The apparatus was placed in an incubator at $37^{\circ} \mathrm{C}$ for 8,24 and $72 \mathrm{~h}$. Control myotubes were maintained under normoxic conditions $(21 \% \mathrm{O} 2+5 \% \mathrm{CO} 2)$ for $8 \mathrm{~h}$. The oxygen tension of the ischaemic media was $6.8 \mathrm{kPa}$ compared to $16.2 \mathrm{kPa}$ in control media. The media was collected and centrifuged at $1600 \mathrm{~g}$ for $5 \mathrm{~min}$ at $4^{\circ} \mathrm{C}$ and stored at $-20^{\circ} \mathrm{C}$. The myotube monolayer was harvested on ice, washed with cold PBS and following this, $75 \mu$ l RIPA buffer $1 \times$ solution $\left(150 \mathrm{mM} \mathrm{NaCl}, 1.0 \%\right.$ IGEPAL ${ }^{\circledR}$ CA-630, $0.5 \%$ sodium deoxycholate, 0.1\% SDS, and 50 mM Tris, pH 8.0; product number R0278; Sigma Aldrich, St Louis, MO, USA) and $75 \mu$ l of Laemmli buffer was added to each well. The monolayer was scrapped with a cell scrapper and collected into a $1.5 \mathrm{~mL}$ Eppendorf tube using a syringe with a $23 \mathrm{G}$ needle. A total of $5 \mu \mathrm{l}(2-5 \%)$ of 2 -mercaptoethanol was added to the cell extract, which was then heated at $95^{\circ} \mathrm{C}$ for 5 min. Extracts were then centrifuged at $1600 \mathrm{~g}$ for $5 \mathrm{~min}$ at $4^{\circ} \mathrm{C}$. The supernatant was collected and stored at $-80^{\circ} \mathrm{C}$ for western blot analysis.

\section{Pre-treatment studies}

To investigate the role of TLR2 and TLR6 and the downstream signalling pathways involved in ischaemia-induced apoptosis and IL-6 induction, the following agonist and neutralising antibodies/inhibitors were used: TLR2/6 agonist PAM2CSK4 $(10 \mu \mathrm{g} / \mathrm{mL}$; EMCMicroCollections, Tuebingen, Germany) was incubated with myotubes for 8h; anti-TLR2 antibody (50 $\mathrm{gg} / \mathrm{mL}$; T2.5; eBioscience, San Diego, USA) and anti-TLR6 antibody $(1.25 \mu \mathrm{g} / \mathrm{mL}$; pab-hstIr6; Invivogen, San Diego, USA) was added to the myotubes $30 \mathrm{mins}$ prior to incubating in ischaemia or added with PAM2CSK4; MyD88 inhibitor $(100 \mu \mathrm{g} / \mathrm{mL}$; tlrl-pimyd; Invivogen) was added $24 \mathrm{~h}$ prior to incubating in ischaemia; TRIF inhibitor (20 $\mu \mathrm{M}$; tIrl-pitrif; 
Invivogen) was added 6h prior to incubating in ischaemia; and NF-KB inhibitor (10 $\mu \mathrm{M}$; Celastrol ant-cls; Invivogen) was added just before incubating in ischaemia (all based on dose-ranging experiments; data not shown).

\section{Immunocytochemistry}

\section{TLR2 and TLR6 co-localisation}

OCT embedded gastrocnemius muscle was cut into $6 \mu \mathrm{m}$ sections and mounted for doubleimmunofluorescence staining. Sections were fixed with ice cold acetone and blocked with $10 \%$ chicken serum. Following this, sections were incubated in rabbit anti-human TLR2 antibody (1:50, sc-10739; Santa Cruz Biotechnology, Santa Cruz, CA, USA) and goat antihuman TLR6 antibody (1:50, sc-5657; Santa Cruz Biotechnology). After incubation with primary antibody or its IgG isotype control for $1 \mathrm{~h}$ at room temperature, slides were washed with PBS and treated with Texas Redchicken Anti-Rabbit (1:200, Invitrogen) and FITCchicken Anti-goat (1:200, Invitrogen) secondary antibodies. The slides were washed with PBS and coverslips were mounted using VECTASHIELD ${ }^{\circledR}$ mounting medium containing DAPI. The images were viewed using a fluorescence microscope (Axioscop 2; Carl Zeiss Microimaging).

\section{Apoptosis}

Cleaved caspase-3 expression was used to assess apoptosis. Chamber slides were removed from the hypoxic chamber and the myotube monolayer was fixed in $4 \%$ formaldehyde for $5 \mathrm{~min}$. Serum block was performed with $10 \%$ goat serum and cells were incubated with Cleaved caspase-3 (Asp175) antibody (1:50 Cell Signalling Technology, Danvers, MA, USA) or its IgG isotype control for $1 \mathrm{~h}$ at room temperature. Slides were then treated with Texas $\operatorname{Red}^{\circledR}$-X goat anti-mouse IgG antibody (Invitrogen), in 1:1000 dilution for $60 \mathrm{~min}$ in the dark at room temperature. The slides were washed with PBS and coverslips were mounted using VECTASHIELD ${ }^{\circledR}$ mounting medium containing DAPI. The images were viewed using a fluorescence microscope.

\section{Western blot analyses}

Equal amounts of denatured protein were loaded per lane $(24 \mu \mathrm{g})$ and were separated on 12\% Tris-glycine gel followed by transfer onto a nitrocellulose membrane (Hybond-C extra; GE Healthcare Life Sciences, Buckinghamshire, UK). The membranes were blocked with 5\% milk in PBS/0.1\% Tween 20 and incubated overnight at $4^{\circ} \mathrm{C}$ with the following antibodies: 
TLR2 (1:500; sc-10739; Santa Cruz), TLR6 (1:500; sc5661; Santa Cruz), TLR6 (1:500; ab-71429; Abcam, Cambridge, UK), NF-KB (1:1000;C22B4; Cell Signalling), Phospho-NF-kB (1:2000; 93H1; Cell Signalling);

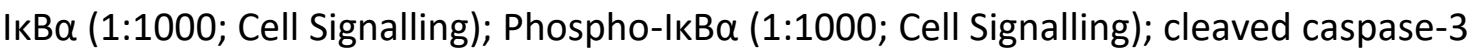
(1:1000; 9661;Cell Signalling); BAX (1:500; sc-526; Santa Cruz), BCL-2 (1:500; sc-783; Santa Cruz), HMGB-1 (1:1000; ab18256; Abcam) and $\beta$-tubulin-loading control (1:10000; ab4074; Abcam). The membranes were then washed and incubated with horseradish peroxidaselinked corresponding secondary antibody at room temperature. The membrane was developed using chemiluminescent substrate (Amersham ECL Plus ${ }^{\mathrm{TM}}$ Western Blotting Detection Reagent; GE Healthcare Life Sciences) and the blots were developed against photographic film (Amersham Hyperfilm ECL, GE Healthcare Life Sciences). Band intensities were determined by densitometry using Biospectrum ${ }^{\circledR} A C$ imaging system (UVP, Upland, CA, USA).

\section{Immunoprecipitation}

Ischaemic and control C2C12 myotube monolayers were lysed with lysis buffer (1\%SDS, $1.0 \mathrm{mM}$ sodium-orthovandate, $10 \mathrm{MM}$ Tris $\mathrm{pH} 7.4$ ). The lysate was than boiled for $5 \mathrm{mins}$, sonicated and centrifuged. $100 \mu$ of lysate was incubated at $4^{\circ} \mathrm{C}$ for $1 \mathrm{~h}$ with $2 \mu \mathrm{g}$ anti-TLR2 (sc10739) or anti-TLR6 (sc5661), 400 $\mu \mathrm{I}_{2} \mathrm{O}$ and $2 \mathrm{X}$ immunoprecipitation buffer (2\% Triton X100, 300mM NaCL, 20mM Tris pH 7.4, 2mN EDTA, 2mM EGTA pH 8.0, 0.4mM

sodiumorthovandate, $0.4 \mathrm{mM}$ PMSF, $1 \% \mathrm{NP}-40$ ). Subsequently the lysate was incubated with $50 \mu$ l protein G-Sepherose (P3296; Sigma-Aldrich, UK) for 30 mins at $4{ }^{\circ} \mathrm{C}$ with agitation.

Bound proteins were than washed twice with $1 \mathrm{X}$ immunoprecipitation buffer and the pellet resuspended in $45 \mu \mathrm{l} 2 \mathrm{X}$ laemlli buffer. Immune complexes were separated by Tris-glycine, transferred to nitrocellulose, and immunoblotted with TLR2 or TLR6 antibodies. Following optimisation of the antibody and technique for the immunoprecipitation studies we did not include IgG control for further experiments. Proteins were visualized by using chemiluminescence followed by exposure to photographic film.

\section{IL-6 Assay}

IL-6 protein concentrations in the culture media were determined using an ELISA kit (Mouse IL-6 ELISA Ready-SET-Go! ${ }^{\circledR}$; eBioscience, San Diego, USA) following the manufacturer's protocol. Samples and known standards were assayed in triplicate. The lower detection limit was $4 \mathrm{pg} / \mathrm{mL}$. 


\section{Statistical Analysis}

Data was analysed with GraphPad Prism software, version 5. Parametric data were compared using a 2-tailed Student's T-test and Analysis Of Variance (ANOVA)/Turkey HSD test, values represent mean $\pm S E$. Non-parametric data were described using median \pm range and were compared using a 2-tailed Mann-Whitney $U$ test. P-value $<0.05$ was considered statistically significant.

\section{Informed Consent Statement}

All procedures followed were in accordance with the ethical standards of the responsible committee on human experimentation (institutional and national) and with the Helsinki Declaration of 1975, as revised in 2000. Written informed consent was obtained from patients with prior approval from the Royal Free \& Medical School Research Ethics Committee (REC Ref: 29-2000).

No animal studies were carried out by the authors for this article

\section{Results}

TLR2 and TLR6 protein expression is upregulated in critically ischaemic muscle and in C2C12 myotubes exposed to simulated ischaemia

TLR1-7 have been shown to be expressed in skeletal muscle (9) and a number of studies have demonstrated that their expression is upregulated following ischaemia in various organ systems. However previous studies have not looked at the effect skeletal muscle ischaemia on TLR expression. We found a significant upregulation of TLR2 ( $P=0.004)$ and 6 $(p=0.002)$ protein expression in muscle from patients with CLI (Figure $1 b \& d)$. During our optimisation of the in vitro ischaemia model we investigated different durations of hypoxia, thus exposing the myotubes to $8 \mathrm{~h}, 24 \mathrm{~h}$ and $72 \mathrm{~h}$. We found that $8 \mathrm{~h}$ was the optimum duration for the myotubes to be exposed to ischaemia in terms of ischaemic parameters such as the $\mathrm{pH}, \mathrm{PO} 2, \mathrm{CO} 2$ and lactate. Longer periods of exposure to hypoxia began to effect the viability of the myotubes. We observed that $8 \mathrm{~h}$ and $24 \mathrm{~h}$ ischaemia did not have a significant effect on TLR2 and TLR6 expression (Data not shown). However myotubes exposed to $72 \mathrm{~h}$ ischaemia showed a significant upregulation of TLR2 $(P=0.006)$ and 6 $(\mathrm{P}=0.0014)$ protein expression (Data not shown).

TLR2 and 6 co-localise and co-immunoprecipitate in muscle following ischaemia with subsequent activation of the TLR signalling pathway 
TLR 2 heterodimerises with TLR1 or 6 depending on the ligand that is presented. We found increased TLR2 and TLR6 co-localisation in ischaemic human muscle (Data not shown) as well as co-immunoprecipitation of TLR2 and TLR6 following ischaemia (Figure 1f). This would suggest that ischaemia leads to TLR2 and TLR6 heterodimerisation and activation of the signalling pathway. In its inactive form NF-KB is bound to the inhibitory complex $\mid \kappa B \alpha$. Following TLR pathway activation, IKB $\alpha$ is phosphorylated and NF-KB is released. Further, optimal NF-KB activation occurs following phosphorylation of the p65 sub-unit of NF-KB. Considering these principles we assessed protein expression of IKB and NF-KB (p65) as well as their phosphorylated forms in control and ischaemic $\mathrm{C} 2 \mathrm{C} 12$ myotubes. Protein expression of both IKB ( $P=0.0028$; Figure $2 a)$ and NF-KB ( $P=0.003$; Figure $2 c)$ was reduced in ischaemic myotubes. Conversely there was significantly increased expression of the phosphorylated form of $1 \kappa B \alpha(P<0.0001$; Figure $2 a)$ and NF-KB ( $P=0.01$; Figure $2 c)$ in ischaemic but not control myotubes. This indicated that simulated ischaemia not only leads to TLR2 and TLR6 heterodimerisation but also activation of the TLR signalling pathway and subsequent NF-KB activation by its phosphorylation.

\section{TLR2 and 6 blockade reduces ischaemia-induced apoptosis}

Increased apoptosis has been demonstrated in skeletal muscle of patients with PAD (1). We therefore evaluated the effect of ischaemia on myotube apoptosis. We found that ischaemia increased apoptosis as shown by increased $B C L 2$-associated $X$ protein (BAX) $(P=0.005$; Figure $3 b)$ and decreased $B$-cell lymphoma 2 ( $B C L 2)(P=0.002$; Figure $3 b$.) expression. This finding was confirmed by increased cleaved caspase-3 expression on western blot (Figure 3d) and immunofluorescence (Figure 3a). Further, neutralising antiTLR2 ( $P<0.01$; Figure 3d) and anti-TLR6 $(P<0.01$; Figure $3 d)$ antibodies reduced ischaemiainduced apoptosis as represented by reduced cleaved caspase-3 protein expression on western blot. These findings suggest that ischaemia-induced apoptosis is mediated via TLR2 and TLR6 activation in skeletal muscle.

\section{Ischaemia-induces skeletal muscle inflammation via TLR2 and TLR6}

Patients with PAD have been reported to have high levels of circulating cytokines such as TNF- $\alpha$ and IL-6 (24) and ischaemia itself is known to trigger the release of inflammatory cytokines in a variety of organs. We found that IL- 6 release was significantly upregulated in the media of the ischaemic myotubes $(P<0.01$; Figure $4 a)$. In addition, the TLR2 and TLR6 agonist PAM2CSK4 also increased IL-6 ( $P<0.01$; Figure 4a) release in the absence of an ischaemic stimuli. Further, neutralising anti-TLR2 $(P<0.01)$ and $6(P<0.01)$ antibodies both reduced the ischaemia-induced IL- 6 release indicating that the IL- 6 release was mediated via the activation of these 2 receptors (Figure $4 a$ ). 


\section{Ischaemia-induced IL-6 generation and apoptosis is mediated via MyD88 signalling pathway and NF-KB activation}

TLR signal transduction is mediated via the MyD88 dependent and MyD88 independent pathways and this leads to the activation of a number of transcription factors such as NF-KB and AP-1. We sought to determine whether both pathways are utilised in ischaemia-induced apoptosis and IL- 6 release and whether IL- 6 generation is a result of NF-KB activation. C2C12 myotubes were pre-treated with a MyD88, TRIF or NF-kB inhibitor prior to exposure to $8 \mathrm{~h}$ simulated ischaemia. The $\mathrm{C} 2 \mathrm{C} 12$ myotubes pre-treated with the MyD88 $(\mathrm{P}<0.01)$ and NF-KB $(P<0.01)$ inhibitors showed reduced IL-6 generation, whilst TRIF inhibition as well the MyD88 and TRIF control peptides did not reduce ischaemia-induced IL-6 release (Figure 4b). Further MyD88 inhibition but not TRIF inhibition reduced ischaemia-induced apoptosis as shown by the reduced cleaved caspase-3 expression (Figure 5a). My88 inhibition disrupted ischaemia-induced NF-KB phosphorylation and hence activation however TRIF inhibition did not abrogate ischaemia-induced NF-KB activation (Figure $5 c$ ).

\section{Potential Endogenous Ligands}

Among the endogenous ligands or damaged associated molecular patterns (DAMPs) that activate TLRs, HMGB-1 and the HSPs have been implicated in activating TLRs following an ischaemic insult. We therefore determined the expression of HMGB-1 and HSPs 60(25) and $70(26)$ in ischaemic $\mathrm{C} 2 \mathrm{C} 12$ myotubes. We have found that following an ischaemic insult there was an increase in the expression of HMGB-1 (P=0.007; Figure 6a), HSPs 60 ( $P=0.0003$; Figure $6 \mathrm{c})$ and $70(\mathrm{P}<0.0001$; Figure $6 \mathrm{c})$

\section{Discussion}

The management of $\mathrm{CLI}$ is pre-dominantly aimed at improving blood flow or in severe cases major amputation is required to prevent progression of gangrene. However despite successful improvement in blood flow to the ischaemic leg many patients still complain of no improvement of symptoms in terms of pain, exercise tolerance and quality of life and therefore deem the surgery to be unsuccessful. The exact pathophysiology as to why these patients have ongoing muscle dysfunction despite the restoration of blood flow is unclear. However emerging evidence suggests that this may be due to ischaemia-induced skeletal muscle damage and it is thought that inflammation plays a central role in this process (27). In support of the theory that inflammation plays an important role in skeletal muscle damage in $\mathrm{CLI}$, it has been shown that raised levels of circulating pro-inflammatory cytokines such as TNF $\alpha$, IL-6 and chemokines are found in patients with PAD (24).

The discovery of TLRs and their role in immunity and inflammation has led to significant advances in terms of understanding the pathophysiology of ischaemia and ischaemia/reperfusion mediated organ injury. In particular TLR2 and TLR4 have been 
strongly implicated in myocardial injury following sustained ischaemia and TLR 2 (28) and TLR4 (29) knockout mice have been shown to have reduced myocardial infarct size. These emerging studies along with the fact TLRs are also expressed on skeletal muscle provide a potential understanding of how ischaemia in CLI patients causes skeletal muscle damage.

Preliminary studies using focussed RT-PCR microarray studies carried out in our lab demonstrated upregulation of gene expression of cell surface TLRs 1, 2, 4 and 6 as well as TLR-related cofactors MyD88 and CD14, and downstream targets IL6, IL8, IRAK1 in muscle from CLI patients. TLR2 upregulation was particularly marked; further, TLR 2 and 6 heterodimerisation had been described (30). Therefore we decided to investigate these 2 TLRs further. In the present study, we have not only shown that TLR2 and TLR6 are expressed on human skeletal muscle but that their expression is increased in ischaemic skeletal muscle. We observed from immunofluorescence staining that TLRs are expressed on endothelium (CD31), neutrophil (CD43), and macrophages (CD68) within muscle tissue. Given this wide spread expression as well as known expression of TLRs on muscle cells, we decided to focus here on the effect of ischaemia on muscle tissue TLR expression, where the TLR activation on individual cell types are likely to exert an autocrine/paracrine effect on the tissue as a whole. In order to understand the significance of this finding we have utilised an in vitro model of simulated ischaemia previously developed by our group. Significantly TLR2 and TLR6 expression was upregulated in the ischaemic $\mathrm{C} 2 \mathrm{C} 12$ myotubes. These findings corroborate with other studies which have shown that ischaemia upregulates TLRs in various tissues both in vivo and in vitro. Our experiments have demonstrated that TLR2 and TLR6 also heterodimerise under ischaemic conditions suggesting ligand binding and activation of the downstream signalling pathway. Indeed we found that IKBa was phosphorylated following an ischaemic insult indicating activation of NFKB which is central to the translocation of NFKB into the nucleus and the subsequent induction of proinflammatory cytokines.

Several studies have shown that following ischaemia cellular death results due to apoptosis and increased apoptosis of skeletal muscle has also been demonstrated in patients with PAD. Our data supports that an ischaemic insult upregulates cellular apoptosis in muscle. We have also shown that ischaemia-induced apoptosis in skeletal muscle is reduced by neutralising anti-TLR2 and TLR6 antibodies indicating that ischaemia-induced apoptosis is mediated via TLR2 and TLR6. The mechanism for this is still poorly understood but studies have shown that TLR2 may promote apoptotic cell death via several pathways such as activation of the JNK-AP-1 (31) pathway or via a pathway involving Fas-associated death domain protein (FADD) and caspase 8 (14).

IL-6 is a potent inflammatory cytokine that plays a significant role in the pathogenesis of cell damage following ischaemia in various tissues. We have established that ischaemia results in an increase in IL- 6 release by myotubes whilst inhibiting TLR2 and TLR6 reduced IL- 6 production. This would suggest that TLR2 and TLR6 are important in the ischaemia-induced 
upregulation of IL- 6 and any subsequent muscle damage propagated by this inflammatory cytokine.

We further sought to elucidate which TLR signalling pathway is implicated in these potentially pathological processes. We found that ischaemia-induced NFKB phosphorylation, apoptosis and IL-6 production in skeletal muscle is mediated via the MyD88 dependent and not the MyD88 independent (TRIF Dependant pathway). The MyD88 pathway may therefore be a potential therapeutic target in reducing ischaemic muscle damage. In addition by targeting a specific pathway, potential side effects of any medical therapy would be kept to a minimum.

Several TLR2 and TLR6 endogenous ligands have been identified that are released in response to stress. In particular HMGB-1, HSPs 60 and 70 have already been implicated in the pathogenesis of renal, liver and myocardial damage following ischaemia. We have shown in our previous studies that there is upregulation in the expression of HMGB-1, HSPS 60 and 70 following ischaemia; further HMGB-1 and HSP 60 have been shown to co-localise with TLR2 and TLR4 $(32,33)$. Therefore, one can speculate that these endogenous ligands may be activating TLR2 and TLR6 and propagating the skeletal muscle damage in patients with limb ischaemia. However, we have yet to identify whether these potential endogenous ligands specifically activate TLR 2 and TLR 6 following skeletal muscle ischaemia or whether there is an alternative mechanism for their activation. Recombinant HMGB-1 has been shown to worsen myocardial injury following I/R whereas treatment with the HMGB-1 antagonist HMGB-1 box A reduced infarct size (11). Thus, it would be interesting to see whether antagonising HMGB-1, HSPs 60 or 70 may reduce skeletal muscle damage following an ischaemic insult.

This study has several limitations: analyses of our muscle biopsies from patients provided only observational evidence for the involvement of TLR2 and TLR6 in ischaemic skeletal muscle. Further investigation was carried out in an in vitro model where $\mathrm{C} 2 \mathrm{C} 12$ myotubes were used. This is an established murine cell line which may not fully reflect the situation in human cells. In vitro models are also not representative of the complex in vivo conditions that occur. However, using a combination of patient tissue analyses and in vitro experiments has allowed us to start elucidating potential pathways for more detailed investigation. As previously mentioned other TLRs have shown to play a role in ischaemia induced damage (34). In particular TLR 2 has also been shown to heterodimerise with TLR1 under certain conditions. It would not be surprising that the other TLRs may be also be playing a role in skeletal muscle injury as the functional 11 TLRs mediate their signalling via the same pathways. Therefore it should be pointed out that whilst we have shown that TLR2 and TLR6 play role in ischaemia induced skeletal muscle damage future studies focusing on the other TLRs in particular TLR4 would be beneficial.

In conclusion we have shown that TLR2 and TLR6 are upregulated in skeletal muscle following ischaemia. The receptors heterodimerise leading to activation of the MyD88 
signalling pathway with subsequent activation of NFKB. This results in upregulation of the inflammatory cytokine IL-6 as well as increase in muscle apoptosis. It is most likely that this results in an environment whereby the inflammatory milieu leads to a myopathy and thus limits the therapeutic effect of revascularisation. Our study has therefore identified potential therapeutic targets such as the MyD88 signalling pathway which may be blocked in order to prevent or reduce muscle damage. It is possible that medical therapy with the aim of reducing the skeletal muscle damage in conjunction with improvement in blood flow may provide symptomatic relief and improve functional outcomes.

\section{Acknowledgements}

We thank the theatre staff of The Heart Hospital and the Royal Free Hospital for helping with tissue collection.

\section{Disclosures}

None.

\section{Funding}

This work was supported by Circulation Foundation research fellowship (fellowship to $\mathrm{H}$. Patel). J. Tsui and S. Shaw were supported by a Royal Society International Joint Project grant.

\section{References}

1. Mitchell RG, Duscha BD, Robbins JL, Redfern SI, Chung J, Bensimhon DR, et al. Increased levels of apoptosis in gastrocnemius skeletal muscle in patients with peripheral arterial disease. Vasc Med. [Multicenter StudyResearch Support, N.I.H., Extramural]. 2007 Nov;12(4):285-90.

2. Cieri E, Lenti M, De Rango P, Isernia G, Marucchini A, Cao P. Functional ability in patients with critical limb ischaemia is unaffected by successful revascularisation. Eur J Vasc Endovasc Surg. 2011 Feb;41(2):256-63.

3. Oresanya L, Zhao S, Gan S, Fries BE, Goodney PP, Covinsky KE, et al. Functional outcomes after lower extremity revascularization in nursing home residents: a national cohort study. JAMA internal medicine. 2015;175(6):951-7. 
4. Alboni S, Cervia D, Ross B, Montanari C, Gonzalez AS, Sanchez-Alavez M, et al. Mapping of the full length and the truncated interleukin-18 receptor alpha in the mouse brain. J Neuroimmunol. [Research Support, N.I.H., Extramural Research Support, Non-U.S. Gov't]. 2009 Sep 29;214(1-2):4354.

5. Pipinos, II, Judge AR, Selsby JT, Zhu Z, Swanson SA, Nella AA, et al. The myopathy of peripheral arterial occlusive disease: Part 2. Oxidative stress, neuropathy, and shift in muscle fiber type. Vasc Endovascular Surg. [Research Support, N.I.H., Extramural Research Support, Non-U.S. Gov't Review]. 2008 Apr-May;42(2):101-12.

6. Vignaud A, Hourde C, Medja F, Agbulut O, Butler-Browne G, Ferry A. Impaired skeletal muscle repair after ischemia-reperfusion injury in mice. Journal of Biomedicine and Biotechnology. 2010;2010.

7. Cluff K, Miserlis D, Naganathan GK, Pipinos II, Koutakis P, Samal A, et al. Morphometric analysis of gastrocnemius muscle biopsies from patients with peripheral arterial disease: objective grading of muscle degeneration. American Journal of Physiology-Regulatory, Integrative and Comparative Physiology. 2013;305(3):R291-R9.

8. Becker RA, Cluff K, Duraisamy N, Mehraein H, Farhoud H, Collins T, et al. Optical probing of gastrocnemius in patients with peripheral artery disease characterizes myopathic biochemical alterations and correlates with stage of disease. Physiological reports. 2017;5(5):e13161.

9. Frost RA, Nystrom GJ, Lang CH. Multiple Toll-like receptor ligands induce an IL-6 transcriptional response in skeletal myocytes. American Journal of Physiology-Regulatory, Integrative and Comparative Physiology. 2006;290(3):R773.

10. Tsan MF, Gao B. Endogenous ligands of Toll-like receptors. Journal of leukocyte biology. 2004;76(3):514.

11. Andrassy M, Volz HC, Igwe JC, Funke B, Eichberger SN, Kaya Z, et al. High-mobility group box1 in ischemia-reperfusion injury of the heart. Circulation. [Research Support, Non-U.S. Gov't]. 2008 Jun 24;117(25):3216-26.

12. Wu H, Chen G, Wyburn KR, Yin J, Bertolino P, Eris JM, et al. TLR4 activation mediates kidney ischemia/reperfusion injury. J Clin Invest. [Research Support, Non-U.S. Gov't]. 2007

Oct;117(10):2847-59.

13. Nakao Y, Funami K, Kikkawa S, Taniguchi M, Nishiguchi M, Fukumori Y, et al. Surfaceexpressed TLR6 participates in the recognition of diacylated lipopeptide and peptidoglycan in human cells. The Journal of Immunology. 2005;174(3):1566.

14. Aliprantis AO, Yang RB, Weiss DS, Godowski P, Zychlinsky A. The apoptotic signaling pathway activated by Toll-like receptor-2. Embo J. [Research Support, U.S. Gov't, P.H.S.]. 2000 Jul 3;19(13):3325-36.

15. Akira S, Takeda K. Toll-like receptor signalling. Nature Reviews Immunology. 2004;4(7):499-

511.

16. Patel H, Shaw SG, Shi-Wen X, Abraham D, Baker DM, Tsui JCS. Toll-Like Receptors in Ischaemia and Its Potential Role in the Pathophysiology of Muscle Damage in Critical Limb Ischaemia. Cardiology Research and Practice. 2012;2012.

17. Zhang J, Wu H, Wang L, Wang $\mathrm{H}$, Zheng $\mathrm{Q}$. TLR2 mRNA upregulation in ischemic lobes in mouse partial hepatic ischemia/reperfusion injury model. J Huazhong Univ Sci Technolog Med Sci. [Research Support, Non-U.S. Gov't]. 2004;24(2):144-6.

18. de Kleijn D, Pasterkamp G. Toll-like receptors in cardiovascular diseases. Cardiovasc Res. [Research Support, Non-U.S. Gov'tReview]. 2003 Oct 15;60(1):58-67.

19. Tsung A, Hoffman RA, Izuishi K, Critchlow ND, Nakao A, Chan MH, et al. Hepatic ischemia/reperfusion injury involves functional TLR4 signaling in nonparenchymal cells. J Immunol. [Research Support, N.I.H., Extramural Research Support, Non-U.S. Gov't]. 2005 Dec 1;175(11):76618.

20. Rusai K, Sollinger D, Baumann M, Wagner B, Strobl M, Schmaderer C, et al. Toll-like receptors 2 and 4 in renal ischemia/reperfusion injury. Pediatr Nephrol. 2010 May;25(5):853-60. 
21. Chong AJ, Shimamoto A, Hampton CR, Takayama H, Spring DJ, Rothnie CL, et al. Toll-like receptor 4 mediates ischemia/reperfusion injury of the heart. J Thorac Cardiovasc Surg. [Research Support, Non-U.S. Gov't Research Support, U.S. Gov't, P.H.S.]. 2004 Aug;128(2):170-9.

22. Leemans JC, Stokman G, Claessen N, Rouschop KM, Teske GJ, Kirschning CJ, et al. Renalassociated TLR2 mediates ischemia/reperfusion injury in the kidney. J Clin Invest. [Research Support, Non-U.S. Gov't]. 2005 Oct;115(10):2894-903.

23. Joshi D, Patel H, Baker DM, Shiwen X, Abraham DJ, Tsui JC. Development of an in vitro model of myotube ischemia. Lab Invest. 2011 Aug;91(8):1241-52.

24. Signorelli SS, Mazzarino MC, Di Pino L, Malaponte G, Porto C, Pennisi G, et al. High circulating levels of cytokines (IL- 6 and TNFalpha), adhesion molecules (VCAM-1 and ICAM-1) and selectins in patients with peripheral arterial disease at rest and after a treadmill test. Vasc Med. 2003;8(1):15-9. 25. Izaki K, Kinouchi $\mathrm{H}$, Watanabe $\mathrm{K}$, Owada $\mathrm{Y}$, Okubo A, Itoh $\mathrm{H}$, et al. Induction of mitochondrial heat shock protein 60 and 10 mRNAs following transient focal cerebral ischemia in the rat. Brain research Molecular brain research. [Research Support, Non-U.S. Gov't]. 2001 Mar 31;88(1-2):14-25. 26. Zou N, Ao L, Cleveland JC, Jr., Yang X, Su X, Cai GY, et al. Critical role of extracellular heat shock cognate protein 70 in the myocardial inflammatory response and cardiac dysfunction after global ischemia-reperfusion. Am J Physiol Heart Circ Physiol. [In VitroResearch Support, N.I.H., Extramural]. 2008 Jun;294(6):H2805-13.

27. Pipinos, II, Swanson SA, Zhu Z, Nella AA, Weiss DJ, Gutti TL, et al. Chronically ischemic mouse skeletal muscle exhibits myopathy in association with mitochondrial dysfunction and oxidative damage. Am J Physiol Regul Integr Comp Physiol. [Research Support, N.I.H., Extramural]. 2008 Jul;295(1):R290-6.

28. Favre J, Musette P, Douin-Echinard V, Laude K, Henry JP, Arnal JF, et al. Toll-like receptors 2deficient mice are protected against postischemic coronary endothelial dysfunction. Arterioscler Thromb Vasc Biol. [Research Support, Non-U.S. Gov't]. 2007 May;27(5):1064-71.

29. Oyama J, Blais C, Jr., Liu X, Pu M, Kobzik L, Kelly RA, et al. Reduced myocardial ischemiareperfusion injury in toll-like receptor 4-deficient mice. Circulation. [Research Support, Non-U.S. Gov't Research Support, U.S. Gov't, P.H.S.]. 2004 Feb 17;109(6):784-9.

30. Park JS, Gamboni-Robertson F, He Q, Svetkauskaite D, Kim JY, Strassheim D, et al. High mobility group box 1 protein interacts with multiple Toll-like receptors. American Journal of Physiology-Cell Physiology. 2006;290(3):C917.

31. Tang SC, Arumugam TV, Xu X, Cheng A, Mughal MR, Jo DG, et al. Pivotal role for neuronal Toll-like receptors in ischemic brain injury and functional deficits. Proc Natl Acad Sci U S A. [Research Support, N.I.H., Intramural]. 2007 Aug 21;104(34):13798-803.

32. Park JS, Gamboni-Robertson F, He Q, Svetkauskaite D, Kim JY, Strassheim D, et al. High mobility group box 1 protein interacts with multiple Toll-like receptors. Am J Physiol Cell Physiol. [Research Support, N.I.H., Extramural]. 2006 Mar;290(3):C917-24.

33. de Graaf R, Kloppenburg G, Kitslaar PJ, Bruggeman CA, Stassen F. Human heat shock protein 60 stimulates vascular smooth muscle cell proliferation through Toll-like receptors 2 and 4 . Microbes Infect. 2006 Jun;8(7):1859-65.

34. Patel H, Shaw SG, Shi-Wen X, Abraham D, Baker DM, Tsui JC. Toll-like receptors in ischaemia and its potential role in the pathophysiology of muscle damage in critical limb ischaemia. Cardiology research and practice. 2012;2012:121237. 


\section{Figure Legends}

Figure 1

(a) Table demonstrating patient characteristics including risk factors for atherosclerotic vascular disease. (b \& d) Representative TLR2 and 6 western blots of control and CLI patient muscle samples. Blot was reprobed for $\beta$-Tubulin expression as control for protein loading. (c \& e) Densitometric quantification of TLR 2 and 6 levels in CLI muscle, values are median and range, ${ }^{*} p<0.01$ compared to control, Mann-Whitney $\cup$ Test, $n=12$. (f) TLR2 heterodimerises with TLR6 following ischaemia.

Figure 2

(a) Representative western blot showing downregulation of $1 \mathrm{~KB} \alpha$ and upregulation of $\mathrm{P}-\mathrm{I} \mathrm{KB} \alpha$ in $\mathrm{C} 2 \mathrm{C} 12$ myotubes exposed to $8 \mathrm{~h}$ of simulated ischaemia compared to control myotubes. Protein loading was consistent as shown by $\beta$-Tubulin levels. (b) Densitometric analyses of western blots, values are means \pm standard error (SE), $n=3,{ }^{*}<<0.05$ unpaired t-Test(c) Representative western blot showing downregulation of NF-KB and upregulation of P-NF-KB in $\mathrm{C} 2 \mathrm{C} 12$ myotubes exposed to $8 \mathrm{~h}$ of simulated ischaemia compared to control myotubes. Protein loading was consistent as shown by $\beta$-Tubulin levels. (d) Densitometric analyses of western blots, values are means $\pm S E, n=3,{ }^{*} p<0.05$ unpaired t-Test.

Figure 3

(a) Immunofluorescence staining for DAPI (blue) and cleaved caspase-3(green) was performed on control and ischaemic myotubes as well ischaemic myotubes incubated with neutralising anti-TLR2 antibody. There is increased cleaved caspase-3 staining in the ischaemic myotubes and significantly reduced cleaved caspase- 3 staining in the ischaemic myotubes with anti-TLR2 antibody. (b) Representative western blot showing downregulation of $B C L 2$ and upregulation of $B A X$ in $C 2 C 12$ myotubes exposed to $72 \mathrm{~h}$ of simulated ischaemia compared to control myotubes. Protein loading was consistent as shown by $\beta$-Tubulin levels. (c) Densitometric analyses of western blot, values are means \pm $\mathrm{SE}, \mathrm{n}=9$. ${ }^{*} \mathrm{p}<0.05$ unpaired t-Test. (d) Representative western blot showing increased cleaved caspase-3 expression following $8 \mathrm{~h}$ simulated ischaemia. Protein loading was consistent as shown by $\beta$-Tubulin levels. (e) Densitometric analyses of western blots, values are means $\pm \mathrm{SE}, \mathrm{n}=3$. $\mathrm{P}<0.0001, \mathrm{ANOVA},{ }^{*}$, denotes ischaemic myotubes; ${ }^{*}$, denotes AntiTLR2 or 6 Antibody + ischaemic myotubes. Turkey HSD test, $P<0.01$ between: * and control myotubes; * and ** myotubes. 
Figure 4

(a) IL-6 generation in C2C12 myotube supernatant was measured by ELISA. Myotubes were exposed to simulated ischaemia for $8 \mathrm{~h}$ with or without anti-TLR2 or 6 antibodies. Values are mean $\pm \mathrm{SE}, \mathrm{n}=9$. $\mathrm{P}<0.0001$, ANOVA. Turkey HSD test: ${ }^{*} \mathrm{P}<0.01$ vs control, ${ }^{*} * \mathrm{P}<0.01$ vs control, $+P<0.01$ vs ischaemia, $++P<0.01$ vs ischaemia. (b) IL-6 generation in C2C12 myotube supernatant was measured by ELISA. Myotubes were pre-treated with specific inhibitors or control peptides and exposed to simulated ischaemia for $8 \mathrm{~h}$. Values are mean $\pm S E, n=9$. $\mathrm{P}<0.0001$, ANOVA. Turkey HSD test: ${ }^{*} \mathrm{P}<0.01$ vs control, ${ }^{*} * \mathrm{P}<0.01$ vs ischaemia, $+\mathrm{P}<0.01$ vs ischaemia.

Figure 5

(a) Representative western blot showing decreased Cleaved Caspase-3 expression. MyD88 inhibition significantly reduced ischaemia-induced Cleaved caspase-3 expression. Protein loading was consistent as shown by $\beta$-Tubulin levels. (b) Densitometric analyses of western blots, values are means $\pm S E, n=9$. $P<0.0001$, ANOVA. Turkey HSD test: ${ }^{*}<<0.01$ vs control, ** $\mathrm{P}<0.05$ vs Ischaemia. (c) Representative western blot showing NF-KB and P- NF-KB expression in $\mathrm{C} 2 \mathrm{C} 12$ myotubes exposed to $8 \mathrm{~h}$ of simulated ischaemia. Protein loading was consistent as shown by $\beta$-Tubulin levels. (d) Densitometric analyses of western blots, values are means $\pm S E, n=6$. $P<0.0001$, ANOVA. Turkey HSD test: NF-kB expression; ${ }^{*} P<0.01$ vs control, ** $\mathrm{P}<0.01$ vs Ischaemia, $\mathrm{P}-\mathrm{NF}-\mathrm{KB}$ expression; * $\mathrm{P}<0.05$ vs control, $* * \mathrm{P}<0.01$ vs Ischaemia.

Figure 6

(a) Representative western blot showing upregulation of HMGB-1 in C2C12 myotubes exposed to $8 \mathrm{~h}$ of simulated ischaemia compared to control myotubes. Protein loading was consistent as shown by $\beta$-Tubulin levels. (b) Densitometric analyses of western blots, values are means $\pm S E, n=6$. ${ }^{*} p<0.05$ unpaired t-Test. (c) Representative western blot showing upregulation of HSPs 60 and 70 in C2C12 myotubes exposed to $8 \mathrm{~h}$ of simulated ischaemia compared to control myotubes. Protein loading was consistent as shown by $\beta$-Tubulin levels. (d) Densitometric analyses of western blots, values are means $\pm S E, n=6 .{ }^{*} p<0.05$ unpaired t-Test.

Supplementary Figure

Hematoxylin and eosin (H\&E) staining confirming differentiation of $\mathrm{C} 2 \mathrm{C} 12$ myoblasts (a) into multinucleated myotubes (b). 
Figures

\begin{tabular}{|c|c|c|c|}
\hline \multicolumn{4}{|l|}{ Figure 1} \\
\hline a & & & \\
\hline & Control(N=12) & $\mathrm{CU}(\mathrm{N}=12)$ & p value \\
\hline Mean age & $67.17 \pm 0.2$ & $70.02 \pm 57$ & NS \\
\hline Mean ABPI & $0.945 \pm 0.07$ & $0.205 \pm 0.13$ & $p<0.05$ \\
\hline Maso Fondeds & 23 & 8.4 & NS \\
\hline Diaboteses Molitus & 4 & 5 & NS \\
\hline Hypertemsion & 8 & $\theta$ & \begin{tabular}{|l|} 
Na \\
\end{tabular} \\
\hline Clyousto Smoting & 6 & 8 & NGS \\
\hline Hepencholostorolemide & $\theta$ & $\theta$ & NGS \\
\hline sisin therapy & 10 & $\theta$ & NS \\
\hline Aspivin therapy & 10 & 11 & NES \\
\hline
\end{tabular}
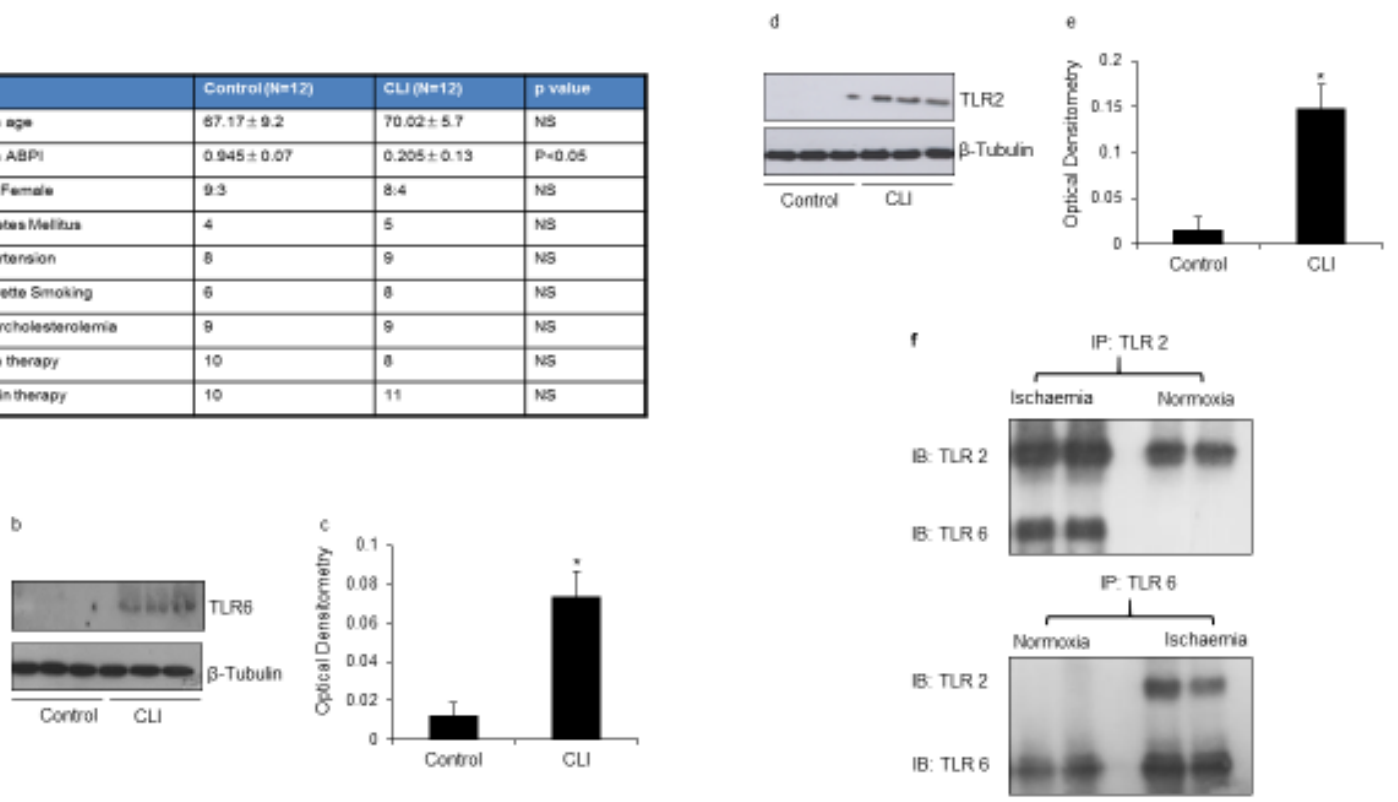

Pigure 2
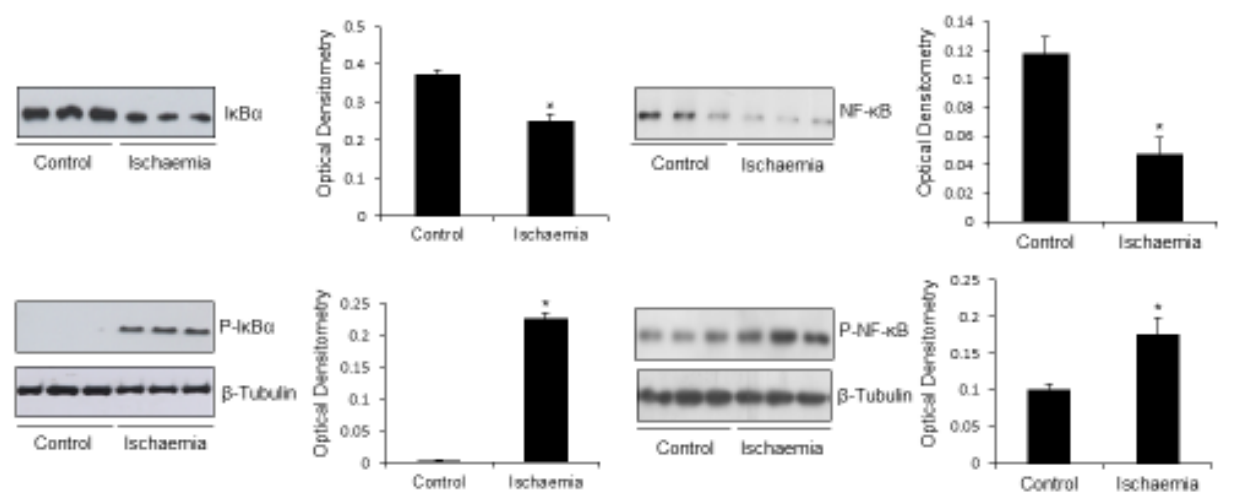


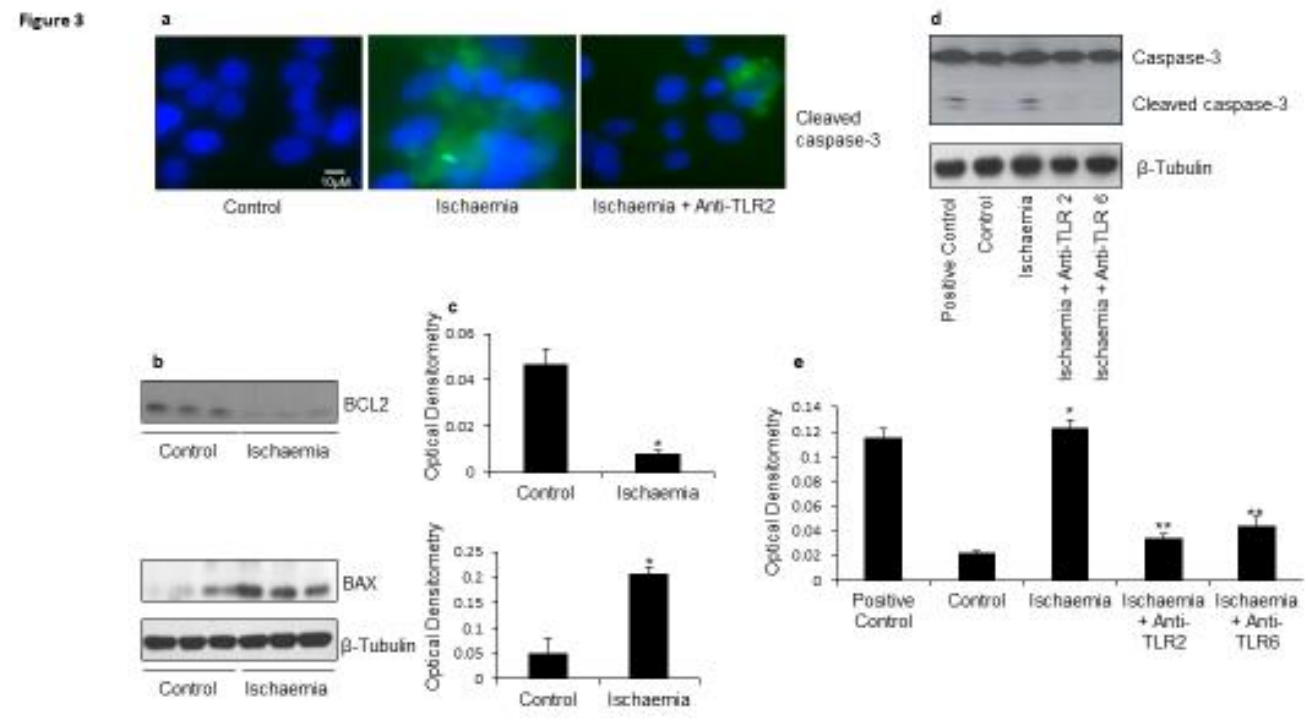

Figure 4
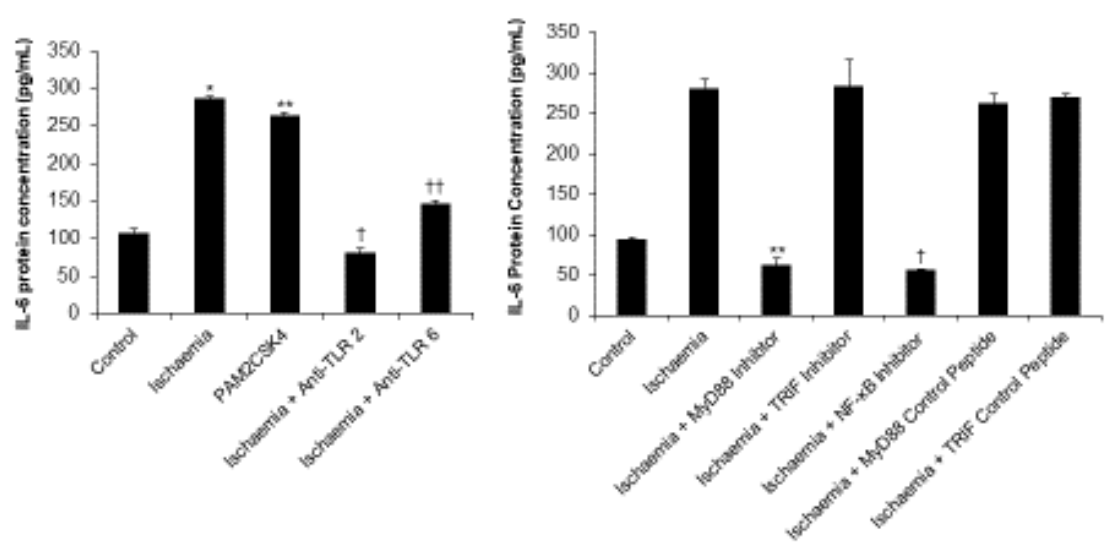
Figure
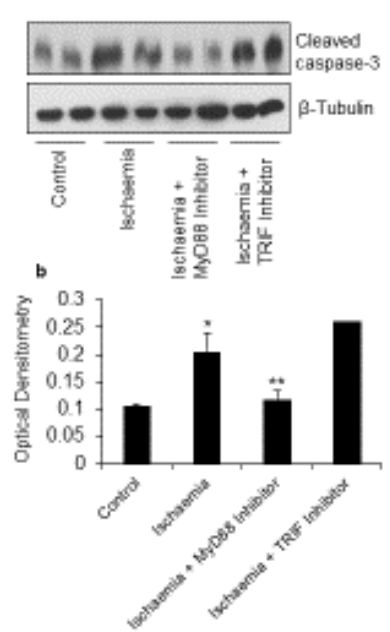
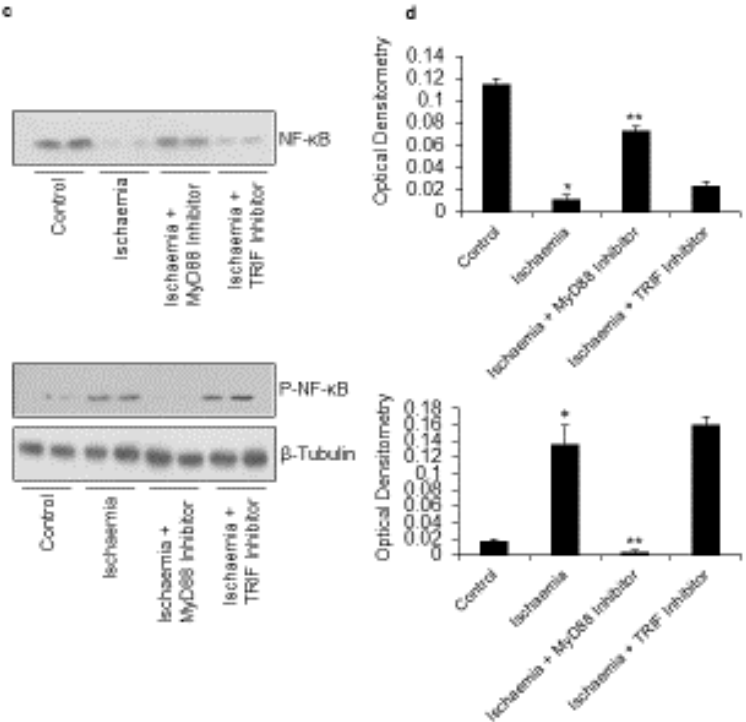

Figure

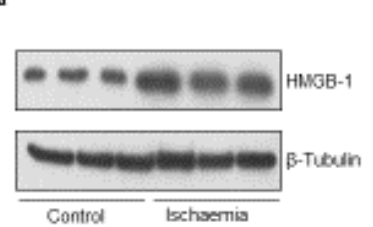

o

d
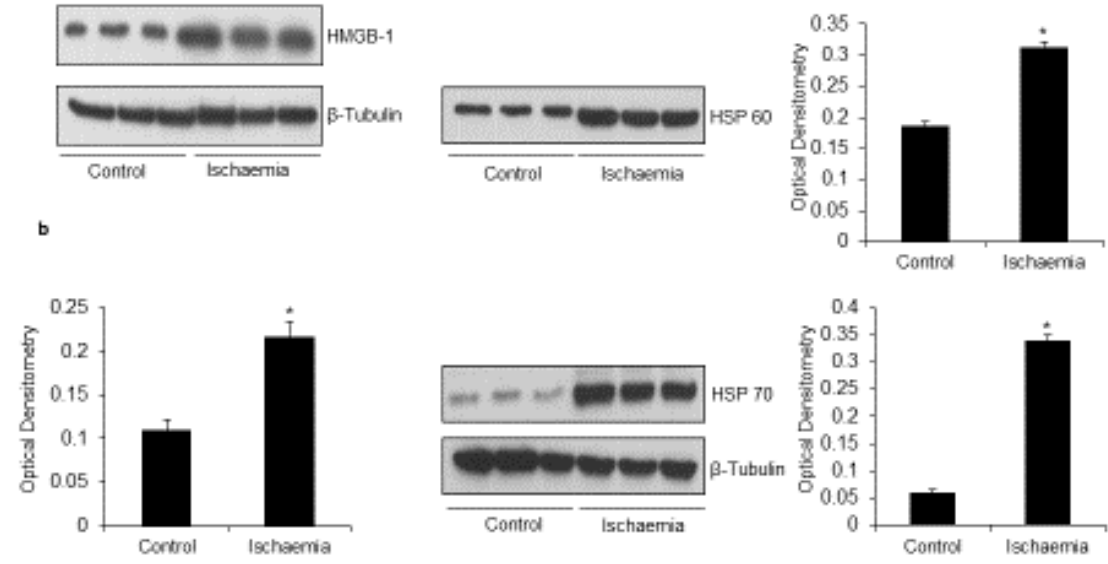